\title{
The Impact of Sex Hormone-Binding Globulin Levels on Thromboembolic Events at Patients with Advanced Stage Adenocarcinoma
}

\section{(1) Mustafa BOZKURT}

Department of Medical Oncology, Acıbadem Mehmet Ali Aydınlar University Faculty of Medicine, Istanbul-Turkey

\begin{abstract}
OBJECTIVE
Several studies have shown that increased risk of venous thromboembolism (VTE) with hormonal contraceptives is mediated through the sex hormone-binding globulin (SHBG) through different pathways. Assuming SHBG as surrogate marker for hormonal stimulus as an increasing risk factor for VTE, we investigated if background SHBG has any impact on VTE in patient with advanced stage adenocarcinoma. Blood drawn from patients with VTE ( $n=45$ ) and from patients without VTE (non-VTE; $n=23$ ), as a control group without a history of VTE, and SHBG levels were compared between groups. There was no difference in SHBG levels between VTE $(59.7 \pm 41.5)$ and non-VTE $(60 \pm 49)(\mathrm{p}=0.71)$ group, but gastric cancer patients had statistically higher SHBG levels $(73.7 \pm 39.9$; range from 36.6 to 176.2$)$ than rest of the cohort (56.54 \pm 44.4 ; range from 9.6 to 246.6 ) ( $\mathrm{p}=0.033$ ), although it was out of scope of this study. Although our study did not show any impact of SHBG levels on risk of VTE at patients with advanced stage adenocarcinoma, it revealed high levels of SHBG at gastric cancer patients which deserve further research.
\end{abstract}

\section{METHODS}

Blood drawn from patients with VTE ( $\mathrm{n}=45$ ) and from patients without VTE (non-VTE; $n=23$ ), as a control group without a history of VTE, and SHBG levels were compared between groups.

\section{RESULTS}

There was no difference in SHBG levels between VTE $(59.7 \pm 41.5)$ and non-VTE $(60 \pm 49)(\mathrm{p}=0.71)$ group, but gastric cancer patients had statistically higher SHBG levels $(73.7 \pm 39.9$; range from 36.6 to 176.2 ) than rest of the cohort (56.54 \pm 44.4 ; range from 9.6 to 246.6$)(\mathrm{p}=0.033)$, although it was out of scope of this study.

\section{CONCLUSION}

Although our study did not show any impact of SHBG levels on risk of VTE at patients with advanced stage adenocarcinoma, it revealed high levels of SHBG at gastric cancer patients which deserve further research.

Keywords: Adenocarcinoma; sex hormone-binding globulin; venous thromboembolism. Copyright $\odot$ 2022, Turkish Society for Radiation Oncology

\section{Introduction}

Sex hormone binding-globulin (SHBG) is a carrier glycoprotein produced in the liver and binds the steroid sex hormones (testosterone and 17 $\beta$-estradiol).[1] SHBG serum levels increase dose dependently on estrogen intake but decrease after the administration of progesterone and can be viewed as an indicator of total estrogenicity.[2] Several studies have shown that increased risk of venous thromboembolism (VTE) with hormonal

\section{Dr. Mustafa BOZKURT}

Acıbadem Mehmet Ali Aydınlar Üniversitesi Tıp Fakültesi,

Tıbbi Onkoloji Anabilim Dalı,

İstanbul-Turkey

E-mail: bozkurtmster@gmail.com 
contraceptives is mediated through the SHBG through different pathways. It has been demonstrated that SHBG levels were positively correlated with acquired activated protein $\mathrm{C}$ (APC) resistance among combined oral contraceptive (COC) users. [3,4] In addition, there is a positive correlation between SHBG and thrombin generation that increases the predisposition for VTE, [5] in the absence of APC resistance among users of hormonal contraceptives.[6] Consecutive evidence suggest that SHBG may be used as a biomarker in assessing prothrombotic profile of hormonal contraception. European Medicines Agency recommends SHBG measurements for the assessment of prothrombotic profile in the development of combined hormonal contraceptive.[7] However, SHBG serum levels are not only determined solely by estrogenicity but also associated with age, gender, body mass index, diabetes, hyperthyroidism, liver disease, and genetic variants of SHBG gene.[8] Recently, researchers identified that high serum SHBG would be a biomarker for early gastric cancer.[9]

SHBG can be easily and rapidly measured in routine laboratories and may be useful to asses prothrombotic profile and further validation with more studies as biomarker in gastric cancer. Assuming SHBG as surrogate marker for hormonal stimulus as an increasing risk factor for VTE, we herein sought to elucidate if background SHBG has any impact on VTE in patient with advanced stage adenocarcinoma.

\section{Materials and Methods}

\section{Patients}

This is a retrospective case-control study including patients 18 years and older with histologic diagnosis of adenocarcinoma followed by Istanbul Bilim University Department of Medical Oncology between October 2007 and April 2012. Among 1845 patients, 99 (5.4\%) experienced VTE. The diagnosis of VTE was confirmed by radiology and the patients were treated accordingly. We defined VTE according to their sites as deep venous thrombosis (DVT), pulmonary embolism (PE), other vascular territories (subclavian, jugular, superior vena cava, and iliac veins) (OVTs), and central venous catheter related (CVC-R). Blood drawn from patients with VTE $(n=45)$ at any time, during active treatment or follow-up and any stage of cancer, and from patients without VTE (non-VTE; $n=23$ ), as a control group without a history of VTE during at least a 29-week duration after cancer diagnosis which is the median time from diagnosis to VTE in our cohort.
SHBG levels were compared between groups. All the laboratory analyses held in the Department of Biochemistry, Haseki Research and Training Hospital. The study protocol approved by the Local Ethics Committee of Haseki Research and Training Hospital, and written informed consent was obtained from all patients before inclusion into the study. The study granted by Tez ve Akademik Calısmalar Danısma ve Izleme Komisyonu, which is research support organization of Haseki Research and Training Hospital.

\section{Assay of SHBG}

Venous blood samples were collected in tubes from the antecubital vein, followed by overnight fasting. The tubes were centrifuged at $4000 \mathrm{rpm}(10 \mathrm{~min})$ to remove the serum. The serum samples were stored at $-80^{\circ} \mathrm{C}$ until analysis SHBG. We used in vitro diagnostic reagent for the quantitative determination of SHBG in human serum by means of chemiluminescent immunoassay on Beckman Coulter Access Systems. (Beckman Coulter, Inc., 250 S. Kraemer Blvd., Brea, CA 92821 U.S.A.). The access SHBG assay is a sequential twostep immunoenzymatic ("sandwich") assay. A sample is added to a reaction vessel along with paramagnetic particles coated with monoclonal anti-SHBG antibody and saline buffer with proteins. After incubation in a reaction vessel, materials bound to the solid phase are held in a magnetic field while unbound materials are washed away. A second monoclonal anti-SHBG antibody conjugated to alkaline phosphatase is added to the reaction vessel. After the second incubation in the reaction vessel, materials bound to the solid phase are held in a magnetic field while unbound materials are washed away. Then, the chemiluminescent substrate Lumi-Phos ${ }^{\star} 530$ is added to the vessel and light generated by the reaction is measured with a luminometer. The light production is directly proportional to the concentration of SHBG in the sample. The amount of analyte in the sample is determined from a stored, multipoint calibration curve. Reference unit was nmol/L.

\section{Statistical Analysis}

Descriptive statistics were reported as percentages and medians with standard deviations. The Chi-square test was used for the comparison of categorical variables. Student's t-test and Mann-Whitney U-test were used for the comparison of means. $\mathrm{P}<0.05$ was considered statistically significant. Statistical analyses were performed with the use of SPSS version 17 (SPSS Inc., Chicago, Illinois, USA). 


\section{Results}

There was no statistically significant difference in demographics variables. Sixty-eight patients, 35 of them women, aged between 37 and 80 are evaluated. At VTE group, 19 (42\%) had PE, 18 (40\%) had (DVT), 4 (9\%) had CVC-R, and 4 (9\%) had OVT. Frequencies of cancer sites for VTE and non-VTE group were colorectal 20 (29\%), gastric 13 (19\%), pancreatic $11(16 \%)$, breast 10 (15\%), lung $6(9 \%)$, and $8(12 \%)$ other (prostatic 3, hepatobiliary 2, unknown primary 2, and ovarian 1) (Table 1). Thirteen (37\%) women were younger than 50 years old and SHBG levels were not different between males (mean \pm SD; 63.6 \pm 53.6$)$ and females $(56.3 \pm 32.4)(\mathrm{p}=0.98)$. There was no difference in SHBG levels between patients experienced VTE $(59.7 \pm 41.5)$ and patients without VTE $(60 \pm 49)(\mathrm{p}=0.71)$, but gastric cancer patients $(\mathrm{n}=13,11$ with a history of VTE) had statistically higher SHBG levels (73.7 \pm 39.9 ; range from 36.6 to 176.2$)$ than rest of the cohort $(\mathrm{n}=55)(56.54 \pm 44.4$; range from 9.6 to 246.6) $(\mathrm{p}=0.033)$, although it was out of scope of this study.

\section{Discussion}

The association between cancer and venous thrombosis was first described in 1823 by Bouillard[10] and in 1865, Trousseau,[11] later coined as Trousseau's syndrome. Almost all patients with active cancer have some degree of the activation of coagulation cascade[12] resulting 4-7-fold increased risk of VTE than general population[13] and making it an important cause of death in patients with cancer.[14] Even after adjusting for stage, cancer patients with thrombosis have 2 -fold increased risk of mortality compared with cancer patients without. $[15,16]$ Patients with adenocarcinoma are generally considered to more prone to developing VTE duo to the high frequency of adenocarcinoma in patients with thrombosis. A large cohort study of lung cancer showed that the risk of VTE was 20-fold higher in lung cancer patients than in general population (standardized morbidity ratio: 20 [14.6-27.4]), and within the cohort, adenocarcinoma was associated 3-fold increased risk compared to patients with squamous cell carcinoma (crude adjusted hazard ratio 3.1, 95\% CI\% 1.4-6.9).[17]

Table 1. Characteristics of patients with adenocarcinoma $(n=68)$ with and without VTE

\begin{tabular}{|c|c|c|c|}
\hline & \multicolumn{2}{|c|}{ VTE } & \multirow[t]{2}{*}{$\mathbf{p}$} \\
\hline & $\begin{array}{c}\text { Yes } \\
n=45\end{array}$ & $\begin{array}{c}\text { No } \\
n=23\end{array}$ & \\
\hline Age & $60.7 \pm 12(37-80)$ & $55.7 \pm 10.2(38-77)$ & 0.095 \\
\hline Female, $n=35$ (51\%) & $\begin{array}{c}n=22 \\
(58.2 \pm 12.6 ; 37-80)\end{array}$ & $\begin{array}{c}n=13 \\
(52.6 \pm 9.9 ; 38-69)\end{array}$ & 0.18 \\
\hline Male, $n=33(49 \%)$ & $\begin{array}{c}n=23 \\
(63.1 \pm 11.2 ;(42-78)\end{array}$ & $\begin{array}{c}n=10 \\
(59.8 \pm 9.6 ; 44-77)\end{array}$ & 0.42 \\
\hline SHBG, $n=68$ & $59.7 \pm 41.5(9.6-246)$ & $60 \pm 49(14-246.6)$ & 0.71 \\
\hline \multicolumn{4}{|l|}{ Adenocarcinoma by sites } \\
\hline Colorectal, n=20 (29\%) & 10 & 10 & \\
\hline Gastric, $n=13$ (19\%) & 11 & 2 & \\
\hline Pancreatic, $\mathrm{n}=11$ (16\%) & 8 & 3 & \\
\hline Breast, $n=10(15 \%)$ & 5 & 5 & \\
\hline Lung, $n=6(9 \%)$ & 6 & 0 & \\
\hline Prostatic, $n=3(4 \%)$ & 2 & 1 & \\
\hline Cholangiocarcinoma, $\mathrm{n}=2$ (3\%) & 1 & 1 & \\
\hline Unknown primary, $\mathrm{n}=2(3 \%)$ & 2 & 0 & \\
\hline Ovarian, $\mathrm{n}=1$ (2\%) & 0 & 1 & \\
\hline \multicolumn{4}{|l|}{ VTE by sites, n (\%) } \\
\hline PE & $19(42)$ & & \\
\hline DVT & $18(40)$ & & \\
\hline CVC-R & $4(9)$ & & \\
\hline OVTs & $4(9)$ & & \\
\hline
\end{tabular}

VTE: Venous thromboembolism; SHBG: Sex hormone-binding globulin; PE: Pulmonary embolism; DVT: Deep venous thrombosis; CVC-R: Central venous catheter related; OVTs: Other vascular territories (subclavian, jugular, superior vena cava, and iliac veins). 
Likewise, emerging evidence let to investigators develop a predictive model for chemotherapy-associated VTE, which citing as very high risk of primary cancer site for stomach and pancreatic adenocarcinoma.[18] Carcinoma mucins, interacting with selections, propagate platelet-rich thrombus without thrombin generation and suggest a fluid-phase coagulation-independent mechanism for Trousseau's syndrome thus provides a rationale for its frequent association with mucin-rich adenocarcinomas.[19,20] However, mechanism of this coexistence needs to be more explored.

Our results demonstrated that SHBG did not differ in patients with adenocarcinoma with VTE and without VTE indicating that hormonal stimulus or background hormonal milieu did not have any impact on increased risk of VTE in patient with advanced stage adenocarcinoma. The plasma levels of SHBG are the sum of multiple stimulatory and inhibitory factors. Women show decreasing level of SHBG between the second and sixth decades followed by a steady increase at around 60 years, a U-shaped trajectory, whereas men show an increasing levels with age.[21] There is now overwhelming evidence that both, estrogen-containing postmenopausal HRT and COCs is associated with clinically important increased risk of VTE, higher the estrogen content higher the risk, dose dependently. [22] At present, clear explanation for the correlation between the changes in SHBG level and thrombin generation and APC resistance induced by COCs does not exist. Hormonal contraceptives, which are metabolized in the liver, might interfere with the synthesis of procoagulant and anticoagulant factors and SHBG, which are produced in the liver.[6] Our results indicate that cancer-related thrombosis process does not have mutual pathways with hormonal mechanisms.

Tissue factor (TF) expression by cancer cells, which is the most likely explanation of procoagulant activity in cancer patients, is shown to be started at early stages carcinogenesis with the activation of MET oncogene. [23] In the Khorana scoring model for chemotherapyassociated VTE, pancreatic cancer cited as a "very highrisk" tumor type[18] which is reported to express high levels of TF.[24] Lately, Hingorani et al.[25] confirmed the hypercoagulability of pancreatic cancer emphasizing role of TF in VTE in advanced adenocarcinoma. In this Phase II trial, patients with advanced stage pancreatic adenocarcinoma were randomly allocated to treatment with pegvorhyaluronidase alfa (PEGPH20), which degrades hyaluronan, with nab-paclitaxel/gemcitabine (PAG) versus nab-paclitaxel/gemcitabine (AG). However, high rate of (43\%) thromboembolic events (TEs) in PAG arm led to exclusion of patients with TE events in PAG arm, andand initiation of prophylactic use of enoxaparin. With the enoxaparin initiation, TE events were $14 \%$ in PAG and $10 \%$ in AG arms.

In a recent study by Cheng et al.,[9] plasma SHBG levels have been identified and suggested as a potential early diagnostic biomarker for gastric cancer. Although it was out of scope of this study, gastric cancer patients had statistically higher SHBG levels $(73.7 \pm 39.9$; range from 36.6 to 176.2$)$ than rest of the cohort ( $56.54 \pm 44.4$; range from 9.6 to 246.6$)(\mathrm{p}=0.033)$. In this case, SHBG needs to be identified if it is the smoke or the fire.

Although to the best of our knowledge, this is the first study sought to elaborate relationship between SGBG and thrombosis in adenocarcinoma, retrospective nature of study inherent limitations. We are aware and acknowledge that caution required interpreting results when a candidate surrogate marker like SHBG is studied, as they can be severely misleading, hence, its levels are affected by many dependent end independent factors. One other limitation of our study relates to the relatively small population sample.

In conclusion, SHBG levels were not different in cancer patients with VTE compared to controls without VTE. Our study cannot totally exclude the possible association between VTE and SHBG levels in gastric cancer.

Acknowledgment: The author thanks to the sponsor of the study, Tez ve Akademik Calismalar Danisma ve Izleme Komisyonu (TACDIC), which is research support organization of Haseki Research and Training Hospital.

Peer-review: Externally peer-reviewed.

Conflict of Interest: All authors declared no conflict of interest.

Ethics Committee Approval: The study was approved by The Haseki Research and Training Hospital Ethics Committee (No: 2, Date: 20/05/2013).

Financial Support: This study has received financial support from Tez ve Akademik Calismalar Danisma ve Izleme Komisyonu (TACDIC), which is research support organization of Haseki Research and Training Hospital.

\section{References}

1. Kuhl H. Comparative pharmacology of newer progestogens. Drugs 1996;51(2):188-215.

2. Hugon-Rodin J, Gompel A, Plu-Bureau G. Epidemiology of hormonal contraceptives-related venous thromboembolism. Eur J Endocrinol 2014;171(6):R221-30. 
3. Raps M, Helmerhorst F, Fleischer K, Thomassen S, Rosendaal F, Rosing J, et al. Sex hormone-binding globulin as a marker for the thrombotic risk of hormonal contraceptives. J Thromb Haemost 2012;10(6):992-7.

4. Odlind V, Milsom I, Persson I, Victor A. Can changes in sex hormone binding globulin predict the risk of venous thromboembolism with combined oral contraceptive pills? Acta Obstet Gynecol Scand 2002;81(6):482-90.

5. Hou H, Ge Z, Ying P, Dai J, Shi D, Xu Z, et al. Biomarkers of deep venous thrombosis. J Thromb Thrombolysis 2012;34(3):335-46.

6. Hugon-Rodin J, Alhenc-Gelas M, Hemker HC, Brailly-Tabard S, Guiochon-Mantel A, Plu-Bureau G, et al. Sex hormone-binding globulin and thrombin generation in women using hormonal contraception. Biomarkers 2017;22(1):81-5.

7. Committee for Medical Products for Human Use (CHMP). Guideline on clinical investigation of steroid contraceptives in women. London: European Medicines Agency; 2005.

8. Stegeman BH, Helmerhorst FM, Vos HL, Rosendaal FR, Van Hylckama Vlieg A. Sex hormone-binding globulin levels are not causally related to venous thrombosis risk in women not using hormonal contraceptives. J Thromb Haemost 2012;10(10):2061-7.

9. Cheng CW, Chang CC, Patria YN, Chang RT, Liu YR, Li FA, et al. Sex hormone-binding globulin (SHBG) is a potential early diagnostic biomarker for gastric cancer. Cancer Med 2018;7(1):64-74.

10. Bouillard JB BS. De l'Obliteration des veines et de son influence sur la formation des hydropisies partielles: consideration sur la hydropisies passive et general. Arch Gen Med 1823;1:188-204.

11. Trousseau A. Phlegmatia alba dolens. Clinique Medicale de l'Hotel-Dieu de Paris. $3^{\text {rd }}$ ed. Paris: J.-B. Bailliere et Fils; 1868. p. 652-94.

12. Lyman GH, Bettigole RE, Robson E, Ambrus JL, Urban $H$. Fibrinogen kinetics in patients with neoplastic disease. Cancer 1978;41(3):1113-22.

13. Lyman GH, Bohlke K, Khorana AA, Kuderer NM, Lee AY, Arcelus JI, et al; American Society of Clinical Oncology. Venous thromboembolism prophylaxis and treatment in patients with cancer: american society of clinical oncology clinical practice guideline update 2014. J Clin Oncol 2015;33(6):654-6.

14. Chew HK, Wun T, Harvey D, Zhou H, White RH. In- cidence of venous thromboembolism and its effect on survival among patients with common cancers. Arch Intern Med 2006;166(4):458-64.

15. Khorana AA, Francis CW, Culakova E, Fisher RI, Kuderer NM, Lyman GH. Thromboembolism in hospitalized neutropenic cancer patients. J Clin Oncol 2006;24(3):484-90.

16. Khorana AA, Francis CW, Culakova E, Kuderer NM, Lyman GH. Thromboembolism is a leading cause of death in cancer patients receiving outpatient chemotherapy. J Thromb Haemost 2007;5(3):632-4.

17. Blom JW, Osanto S, Rosendaal FR. The risk of a venous thrombotic event in lung cancer patients: higher risk for adenocarcinoma than squamous cell carcinoma. J Thromb Haemost 2004;2(10):1760-5.

18. Khorana AA, Kuderer NM, Culakova E, Lyman GH, Francis CW. Development and validation of a predictive model for chemotherapy-associated thrombosis. Blood 2008;111(10):4902-7.

19. Varki A. Trousseau's syndrome: multiple definitions and multiple mechanisms. Blood 2007;110(6):1723-9.

20. Shao B, Wahrenbrock MG, Yao L, David T, Coughlin $\mathrm{SR}, \mathrm{Xia}$ L, et al. Carcinoma mucins trigger reciprocal activation of platelets and neutrophils in a murine model of Trousseau syndrome. Blood 2011;118(15):4015-23.

21. Maggio M, Lauretani F, Basaria S, Ceda GP, Bandinelli S, Metter EJ, et al. Sex hormone binding globulin levels across the adult lifespan in women--the role of body mass index and fasting insulin. J Endocrinol Invest 2008;31(7):597-601.

22. van Hylckama Vlieg A, Middeldorp S. Hormone therapies and venous thromboembolism: where are we now? J Thromb Haemost 2011;9(2):257-66.

23. Boccaccio C, Sabatino G, Medico E, Girolami F, Follenzi A, Reato $G$, et al. The MET oncogene drives a genetic programme linking cancer to haemostasis. Nature 2005;434(7031):396-400.

24. Nitori N, Ino Y, Nakanishi Y, Yamada T, Honda K, Yanagihara $\mathrm{K}$, et al. Prognostic significance of tissue factor in pancreatic ductal adenocarcinoma. Clin Cancer Res 2005;11(7):2531-9.

25. Hingorani SR, Zheng L, Bullock AJ, Seery TE, Harris WP, Sigal DS, et al. HALO 202: Randomized phase ii study of PEGPH20 plus nab-paclitaxel/gemcitabine versus nab-paclitaxel/gemcitabine in patients with untreated, metastatic pancreatic ductal adenocarcinoma. J Clin Oncol 2018;36(4):359-66. 